\title{
Molecular Cloning of Dormancy-associated MADS-box Gene Homologs and Their Characterization during Seasonal Endodormancy Transitional Phases of Japanese Pear
}

\author{
Benjamin Ewa Ubi \\ National Institute of Fruit Tree Science, Tsukuba, Ibaraki 305-8605, Japan and Biotechnology \\ Research and Development Centre, Ebonyi State University, Abakaliki, P.M.B. 053, Ebonyi State, \\ Nigeria \\ Daisuke Sakamoto \\ National Institute of Fruit Tree Science, Tsukuba, Ibaraki 305-8605, Japan
}

Yusuke Ban

National Institute of Fruit Tree Science, Higashihiroshima, Hiroshima 739-2494, Japan

Takehiko Shimada, Akiko Ito, and Ikuko Nakajima

National Institute of Fruit Tree Science, Tsukuba, Ibaraki 305-8605, Japan

Yoshihiro Takemura and Fumio Tamura

Tottori University, Tottori, Tottori 680-8550, Japan

Toshihiro Saito and Takaya Moriguchi ${ }^{1}$

National Institute of Fruit Tree Science, Tsukuba, Ibaraki 305-8605, Japan

\begin{abstract}
Additional Index words. bud, budbreak, expression, DNA methylation, low temperature requirement
Abstract. To understand the role of the MIKC-type dormancy-associated MADS-box $(D A M)$ genes in the regulation of endodormancy in japanese pear (Pyrus pyrifolia), we isolated two DAM genes from 'Kosui' and characterized their expression throughout the seasonal endodormancy phases in 'Kosui', as well as in TP-85-119 taiwanese pear $(P$. pyrifolia), which is a less dormant type. Several copies of the corresponding $D A M$ genes are present in the $P$. pyrifolia genome. Rapid amplification of cDNA ends enabled the isolation of two full-length cDNAs, designated as PpMADS131 and PpMADS13-2, with complete open reading frames encoding 227 and 234 amino acids, respectively. Multialignment of the two 'Kosui' and the database DAM genes (based on the deduced amino acid sequences) showed that PpMADS13-1 and PpMADS13-2 were highly identical to the Rosaceae DAM genes and encoded the conserved domains characteristic of other MIKC-type MADS-box genes. The phylogenetic relationships showed that PpMADS13-1 and PpMADS13-2 were more closely related to the Prunus DAM, though they formed a unique subclade. The specific expression analysis of $P$ PMADS13-1 and $P$ PMADS13-2 by real-time polymerase chain reaction showed that both $D A M$ genes are gradually down-regulated concomitant with endodormancy breaking. PpMADS131 and $P$ PMADS13-2 showed similar fluctuations in expression patterns, although $P$ pMADS13-2 was more highly expressed relative to PpMADS13-1. The expression of PpMADS13-1 and PpMADS13-2 in the less dormant taiwanese pear, TP-85-119, was quite low (nearly zero level), which is consistent with a down-regulated pattern of expression of the DAM genes in japanese pear, peach (Prunus persica), and japanese apricot (Prunus mume). Differential genomic DNA methylation patterns detected in PpMADS13-1 and PpMADS13-2 were not concomitant with seasonal endodormancy transition phases, suggesting that DNA methylation in these loci under investigation may not be linked to endodormancy progression in 'Kosui'.
\end{abstract}

Perennial deciduous plants possess a distinct feature of being able to recurrently suspend and resume growth in response to seasonal and environmental conditions such as photoperiod and low temperatures. This inability to initiate growth from meristematic structure (and other organs and cells with the capacity for growth resumption), referred to as dormancy, is

Received for publication 21 Oct. 2009. Accepted for publication 4 Feb. 2010. We gratefully acknowledge The Japanese Society for the Promotion of Science for awarding a visiting research fellowship to B.E.U. In addition, this work was partially supported by a grant from the NARO to T.M.

${ }^{1}$ Corresponding author. E-mail: takaya@affrc.go.jp. a complex process that is necessary for plant survival in an unfavorable environment (Rohde and Bhalerao, 2007).

Lateral buds formed in the early summer season enter a paradormant state due mainly to apical dominance. Toward autumn, the control of bud growth inhibition shifts to the bud itself and these dormant structures are referred to as being endodormant (Lang, 1987). The removal of terminal buds or defoliation does not restore the plants' capacity to emerge from endodormancy. A certain amount of chilling accumulation is required for the transition of endodormant buds to an ecodormant state from which buds are capable of growth resumption (dormancy breaking) under favorable environmental conditions 
(Faust et al., 1997; Rohde and Bhalerao, 2007; Yamane et al., 2008). Unlike endodormancy, which is genetically controlled, ecodormancy is provoked by limitations in environmental factors (mainly cold stress) that induce critical signals for bud growth inhibition (Crabbe and Barnola, 1996; Horvath et al., 2003; Yamane et al., 2008).

The commercial production of temperate fruit trees in warmer areas is hindered by a lack of sufficient chilling hours. This has often necessitated the use of chemicals or other physical means whose efficacy and level of phytotoxicity varies with the depth of endodormancy (Keilin et al., 2007). Furthermore, the chilling requirement for endodormancy breaking is potentially hampered by the recent environmental perturbations associated with global warming, leading to serious problems such as trees with barren branches or slow-growing shoots, and irregular or loss of flowering with its resultant effect on productivity (Arora et al., 2003; Sugiura et al., 2007). A search for internal factors or environmental cues controlling endodormancy is therefore of the utmost importance to overcome the problem associated with low chilling requirement for fruit production.

Mutants exhibiting continuous growth in winter have been identified by genetic studies in woody perennial species such as Corylus avallana (Thompson et al., 1985) and peach (Rodriguez et al., 1994). Of these, the evergrowing peach mutant (previously known as evergreen, USDA PI442380), a nondormant genotype identified from southern Mexico, is the best-described mutant (Rodriguez et al., 1994; Thompson et al., 1985; Werner and Okie, 1998). The evergrowing peach trees fails to respond to winter dormancy cues and maintains continuous growth of the terminal apices and a lack of leaf abscission during the prevailing short days and low temperatures until killed by freezing temperatures (Bielenberg et al., 2004; Wang et al., 2002). Inheritance studies have indicated that the nondormant evg trait is controlled by a single recessive nuclear locus (Rodriguez et al., 1994; Wang et al., 2002). Genetic and molecular approaches have led to the identification of putative genes regulating bud endodormancy, among which MIKC-type $M A D S$ box genes were identified as strong candidate genes for regulating endodormancy breaking and were designated as dormancy-associated MADS-box (DAM) genes (Bielenberg et al., 2008). It has been reported that the expression of $D A M$ genes is lost in the evergrowing peach mutant and decreased toward endodormancy breaking in wild peach (Bielenberg et al., 2004, 2008; Jiménez et al., 2009). Recent studies have also shown that $D A M$ genes are differentially expressed in response to seasonal dormancy transitions in other plant systems, including raspberry (Rubus idaeus), japanese apricot, leafy spurge (Euphorbia esula), and peach (Horvath et al., 2008; Li et al., 2009; Mazzitelli et al., 2007; Yamane et al., 2008).

Knowledge of regulation of endodormancy in japanese pear is of the utmost importance to provide the basis for manipulation of the genes controlling endodormancy through genetic and/or transgenic approaches toward a more stable and economic production of this important fruit tree. In this study, we report the molecular cloning of two DAM genes, PpMADS13-1 and $P$ PMADS13-2, in the SVP/AGL24 gene family in japanese pear and we analyzed their expression patterns in the lateral leaf buds through seasonal dormancy transitions. In addition, DNA methylation has been reported to play an important regulatory role in floral differentiation of plants (Dennis and Peacock, 2007; Henderson and Dean, 2004). In particular, it was reported that vernalization and demethylation of DNA are associated with promotion of flowering in Arabidopsis thaliana (Finnegan et al., 2000; Genger et al., 2003). Therefore, we further investigated the role of epigenetic control in the PpMADS131 and PpMADS13-2 loci involving differential genomic DNA methylation through the seasonal dormancy transitional phases. This study suggested the involvement of $D A M$ genes in endodormancy breaking through a down-regulated expression pattern concomitant with the seasonal endodormancy transition phases in the lateral leaf buds. Epigenetic control involving differential genomic DNA methylation of PpMADS13-1 and PpMADS13-2 is not associated with the seasonal endodormancy phases in japanese pear. To the best of our knowledge, this is the first report on the cloning and characterization of $D A M$ genes in pear.

\section{Materials and Methods}

Plant materials. Samples were collected from 34-year-old trees of 'Kosui' japanese pear and 20-year-old trees of TP-85119 , a less-dormant taiwanese pear type (included to confirm the physiological role of the pear $D A M$ genes) grown at the orchard of the National Institute of Fruit Tree Science, Tsukuba, Japan (lat. $36^{\circ} \mathrm{N}$, long. $140^{\circ} \mathrm{E}$ ). Lateral leaf buds collected at intervals throughout the seasonal dormancy transitional phases, from early Sept. 2008 to mid-Feb. 2009 [i.e., 2008 (9 Sept., 21 Oct., 12 Nov., 9 Dec., and 24 Dec.) and 2009 ( 8 Jan. and 12 Feb.)], were used in this study to investigate the expression of the DAM genes in 'Kosui'. The lateral leaf buds of TP-85-119 were collected from mid-November to mid-Dec. 2008 (i.e., 12 Nov. and 9 Dec. 2008). In this study, lateral leaf buds were used instead of floral buds to avoid the confounding effect of floral identity genes on the DAM genes as has been highlighted by Yamane et al. (2008). The dormancy status of the field-grown trees during each of these collection dates was estimated based on the evaluation of cut branches. For the evaluation of endodormancy status in the lateral leaf buds, five current elongating branches with lengths of about $60 \mathrm{~cm}$ and bearing predominantly leaf buds were taken at each collection date and placed in distilled water contained in $500-\mathrm{mL}$ vials in an incubator kept at $25 \pm 1.0{ }^{\circ} \mathrm{C}$ under cool white fluorescent light and a 16-h photoperiod for the forcing treatment. The distilled water in the vials was changed at 2- to 3-d intervals. The endodormancy status (as percentage budbreak or sprouting ratio) at each collection date, which is defined as the stage at which green tissue becomes visible under the bud scales, was recorded based on 5 to 9 buds per branch averaged over five branches scored at $21 \mathrm{~d}$ after incubation. The collected lateral leaf buds were immediately frozen in liquid nitrogen and stored at $-80{ }^{\circ} \mathrm{C}$ until needed for RNA and/or DNA extraction.

ISOLATION OF PARTIAL DAM GENE FRAGMENT FROM 'KOSUI'. Total RNA was isolated from 'Kosui' lateral vegetative buds collected toward the end of the 2008 growing season (21 Oct., which coincided with $0 \%$ sprouting ratio) using a hot borate extraction procedure (Wan and Wilkins, 1994). First-strand cDNA was synthesized from $1 \mu \mathrm{g}$ of total RNA using a SMART RACE cDNA Amplification Kit (Clontech, Palo Alto, CA) and was then used as template for homology-based amplification of the $D A M$ gene fragments. The reverse transcriptase (RT)-polymerase chain reaction (PCR) reaction was performed with the forward ( $5^{\prime}$-GCAGCATCCCAACTCTTTCT-3') and reverse $\left(5^{\prime}\right.$-ATGGGATTGCAAGGTACAGC-3') primers 
designed on the basis of Ppdam6 (GenBank accession no. DQ863252) using the Primer 3 software (Rozen and Skaletsky, 2000) and used for the initial amplification. The RT-PCR was performed in a total volume of $50 \mu \mathrm{L}$ containing $125 \mathrm{ng}$ of cDNA, $500 \mu \mathrm{M}$ dNTPs, $375 \mu \mathrm{M} \mathrm{MgSO}_{4}, 3.125 \mu \mathrm{M}$ of each primer, $0.4 \mathrm{U}$ of KOD Plus polymerase, and $0.1 \times \mathrm{KOD}$ buffer (Toyobo, Osaka, Japan). Following a pre-PCR heating at $95^{\circ} \mathrm{C}$ for $2 \mathrm{~min}$, a cycling profile of $94^{\circ} \mathrm{C}$ for $30 \mathrm{~s}, 50{ }^{\circ} \mathrm{C}$ for $30 \mathrm{~s}$, and $72{ }^{\circ} \mathrm{C}$ for $4 \mathrm{~min}$ was repeated 35 times. A final extension for 10 min at $72{ }^{\circ} \mathrm{C}$ was performed. The 609 -bp product was cloned into the $\mathrm{pCR}^{\circledR}$-Blunt vector using a Zero Blunt ${ }^{\circledR}$ PCR Cloning Kit (Invitrogen, Foster City, CA), sequenced, and annotated to confirm its homology to $D A M$ genes from other plant species (data not shown). This 609-bp fragment was designated as pPpMADS13\#2.

SOUTHERN BLOT ANALYSIS TO DETERMINE THE COPY NUMBER OF THE DAM GeNeS IN THE PEAR GENOME. To estimate the copy number of the $D A M$ genes in the japanese pear genome, as well as understand whether the DAM genes are present in the taiwanese pear genome, genomic DNA was extracted from fresh flower bud tissues of 'Kosui', 'Gold Nijisseiki' ( $P$. pyrifolia), 'Hosui' ( $P$. pyrifolia), and TP-85-119 according to a crude nuclear extraction method (Thomas et al., 1993). Genomic DNA $(10 \mu \mathrm{g})$ was digested at $37^{\circ} \mathrm{C}$ overnight with the restriction enzymes (EcoRI and HindIII). These enzymes were selected because they lacked cleavage sites in the probe region. The resulting restricted fragments from EcoRI and HindIII digests were size fractionated in a $0.8 \%(\mathrm{w} / \mathrm{v}) \mathrm{SeaKem}^{\circledR} \mathrm{GTG}^{\circledR}$ agarose gel (Cambrex BioSciences, Rockland, ME) using 0.8\% $\mathrm{TAE}$ as an electrophoresis running buffer. The fractionated, digested DNA was blotted onto nylon membranes (Hybond-N; Amersham Biosciences, Piscataway, NJ). The 609-bp $p P p M A D S 13 \# 2$ was used to generate the hybridization probe using a DIG DNA Labeling Kit (Roche, Mannheim, Germany). Prehybridization ( $1 \mathrm{~h}$ ) and hybridization (overnight) were done using a high SDS hybridization buffer containing $5 \times \mathrm{SSC}, 50 \%$ formamide, $50 \mathrm{~mm} \mathrm{NaH}{ }_{2} \mathrm{PO}_{4}+\mathrm{Na}_{2} \mathrm{HPO}_{4}(\mathrm{pH} 7.0)$, 2\% blocking reagent (Roche), $0.1 \% \mathrm{~N}$-lauroylsarcosine, and $7 \% \mathrm{SDS}$ at $42{ }^{\circ} \mathrm{C}$. After hybridization, the membranes were washed twice at $65{ }^{\circ} \mathrm{C}$ for $15 \mathrm{~min}$ at moderate stringency $(1 \times \mathrm{SSC}$ and $0.1 \%$ SDS). The detection was performed according to the manufacturer's protocol using a DIG-CSPD system (Roche), and the membranes were exposed to X-ray film (Fuji Film, Tokyo).

Isolation of the FUll-length PPMADS13-1 AND PpMADS13-2 ClONes From 'Kosui'. To isolate the full-length cDNAs of the pear $D A M$ genes, $5^{\prime}$ - and $3^{\prime}$-rapid amplification of cDNA ends (RACE) were performed. A $1-\mu \mathrm{g}$ aliquot of total RNA from 'Kosui' japanese pear was used to prepare 5' -and 3'-RACE-Ready cDNAs with a SMART RACE cDNA Amplification Kit (Clontech) according to the manufacturer's instructions. The $5^{\prime}-$ and $3^{\prime}$-RACE were carried out with $5^{\prime}-$ and $3^{\prime}$-specific primers (first 5'-RACE MADS13: 5' -CCTCGGGC AGATAACATCACCATCCT-3', second 5'-RACE MADS13: 5'-ACCTTGCAATCCCATCCTTGGTACTGG-3', and 3' RACE MADS13: 5'-ATGGTGGAGAAGAAGGCGTGACAT CTG-3') designed based on the initial 609-bp pPpMADS13\#2 sequence and the preceding RACE PCR (for the second $5^{\prime}$ RACE). The cDNA ends were ligated into $\mathrm{pCR}^{\circledR} 2.1$ (Invitrogen) and sequenced. The resultant sequences were aligned to obtain a full-length cDNA sequence. Based on the full-length cDNA sequence information, forward ( $5^{\prime}$-GCAGCATCCCAACTCTT TCT-3') and reverse (5'-CACAGAAATGCCTTCCGATT-3') primers were designed to clone the full-length $D A M$ genes by RT-PCR using the amplification conditions as described for isolation of the partial fragment. The full-length genes were designated as PpMADS13-1 and PpMADS13-2, and have been deposited in the DNA Data Bank of Japan (DDBJ) under accession numbers AB504716 and AB504717, respectively.

Analysis of CDNA SEQuences AND CONSTRuCtion OF PHYLOGENETIC TREE. The full-length PpMADS13-1 and PpMADS13-2 cDNA sequences were used to search homologous sequences via tBLASTx in National Center for Biotechnology Information (NCBI). Multiple alignments of the predicted full-length amino acid sequences were performed between 'Kosui' $D A M$ genes and those of other plant species using ClustalW (Thompson et al., 1994). A phylogenetic relationship tree was then constructed by the neighbor-joining (NJ) method. The NJ method was chosen because it is known to be quite efficient in obtaining reliable trees from large data sets (Zhang and Nei, 1996). Typical motifs of the DAM genes that are conserved among 'Kosui' and other plant species were analyzed via ScanProsite (de Castro et al., 2006).

REAL-TIME QUANTITATIVE RT-PCR. Total RNA was isolated from the lateral leaf bud samples of 'Kosui' and the less dormant taiwanese pear, TP-85-119, was collected as detailed above using a modified hot borate extraction method (Wan and Wilkins, 1994). The synthesis of the first-strand cDNA was made using the SuperScript ${ }^{\top M}$ III First Strand Synthesis System (Invitrogen). The 5.0- $\mu \mathrm{g}$ aliquot of total RNA used in the reaction was first treated with DNase (Promega, Madison, WI) and was reverse-transcribed using SuperScript III Oligo (dT) 20 Primers according to the manufacturer's instructions (Invitrogen). For quantitative real-time PCR, specific primers for PpMADS13-1 [forward: 5'-ACCCACGCTTCATCAGC TACT-3' and reverse: 5'-CCAATTTTTCTAACTTCTG TAGTTCACC-3'; product size of 149 bp] and PpMADS13-2 [forward: 5' -AAATCACGCTTCACCAACTGC-3' and reverse: 5' -CACCAATTTTTCTAACTTGTTCAGTTGATA3'; product size of 152 bp] were designed using the Primer Express software. As an internal control, the pear actin gene was used for the amplification of 'Kosui' and taiwanese pear cDNAs using the actin-specific primers (forward: 5'-CAGG CATTCACGAGACCACA-3' and reverse: 5'-TGCCAG GAACATGGTAGAA-3') to obtain the relative gene expression levels of PpMADS13-1 and PpMADS13-2. The real-time quantification of the first-strand cDNA was performed on the Light Cycler 2.0 System (Roche) and was analyzed with the Light Cycler Software, version 4 . The reaction mixture $(15 \mu \mathrm{L})$ contained $2.0 \mu \mathrm{L}$ of cDNA sample (equivalent to $118 \mathrm{ng}$ of the total RNA), 3.0 $\mu \mathrm{M}$ of each primer, and $7.5 \mu \mathrm{L}$ of SYBR ${ }^{\circledR}$ Green Premix ExTaq II (Takara, Shiga, Japan). For a control reaction, no template was added to the reaction mixture, resulting in no detectable fluorescence signal from the reaction. The PCR conditions were set as follows: initial denaturation for $20 \mathrm{~s}$ at $95{ }^{\circ} \mathrm{C}$, followed by 55 cycles of denaturation at $95{ }^{\circ} \mathrm{C}$ for $5 \mathrm{~s}$, annealing for $20 \mathrm{~s}$ at $60^{\circ} \mathrm{C}$, and extension for $72^{\circ} \mathrm{C}$ at $15 \mathrm{~s}$. Each reaction was subjected to melting-point analysis to confirm single amplified products. Three replications were carried out for each gene. The specificity of each amplification reaction for a given primer set was verified in three ways: 1) by the melt gradient in which fluorescence decreases at a single discrete temperature, indicating the separation of both strands of a single DNA species; 2) by the specific restriction enzyme digestion analysis of the amplified products using XhoI and Pst I, which 
can restrict only the amplified sequence of PpMADS13-1 and PpMADS13-2, respectively; and 3) by the simultaneous analysis of the plasmids of the respective genes using each primer set resulting in marked differences in the absolute quantification values from the respective standardized plasmids (templates) at a given concentration. Transcript levels were estimated using the established standard curve based on the dilution series of the plasmid and these levels were normalized against the actin transcript level in each sample, establishing a relative expression value.

Methylation status of PpMads13-1 and PpMAdS13-2 DURING ENDODORMANCY TRANSITION PHASES. To investigate the genomic DNA methylation status, genomic DNA was extracted as described above from the vegetative buds of 'Kosui' collected at the different seasonal dormancy transition stages from 2008 (21 Oct., 12 Nov., 9 Dec., and 24 Dec.) and 2009 (8 Jan. and 12 Feb.). Genomic DNA (10 $\mu \mathrm{g})$ was digested with the methylation-sensitive isoschizomer pair HpaII/MspI. HpaII and $M s p$ I have multiple cleavage sites in the $D A M$ genes. The resulting restricted fragments from $H p a \mathrm{II}$ and $M s p \mathrm{I}$ digests were size fractionated and blotted onto nylon membranes as described above. To understand the methylation status of PpMADS13-1 and PpMADS13-2 loci, a common reverse primer (5' -GGAAGCCCCAGTTTGAGAGAC-3') and genespecific forward primers for PpMADS13-1 (5'-ATCAAGAT CAGAAAGATCGAC-3'; 647-bp) and PpMADS13-2 (5' ATGGTGAAAAGGATGAATGAG-3'; 701-bp) were used to generate specific PpMADS13-1 and PpMADS13-2 DIGlabeled probes from the coding region. Prehybridization $(1 \mathrm{~h})$ and hybridization (overnight) were done as described above. After hybridization, the membranes were washed twice at $65^{\circ} \mathrm{C}$ for $15 \mathrm{~min}$ at high stringency $(0.5 \times \mathrm{SSC}$ and $0.1 \% \mathrm{SDS})$. The detection was performed as described above.

\section{Results}

ISOLATION OF PARTIAL DAM GENE FRAGMENT FROM 'KoSUI'. To isolate a partial cDNA fragment of the $D A M$ genes from 'Kosui', RT-PCR was performed using primers conserved among the $P$. persica DAM family members and were designed on the basis of Ppdam6 (GenBank accession no. DQ863252). The $\approx 609$-bp PCR product obtained was cloned into the $\mathrm{pCR}^{\circledR}$ Blunt vector using a Zero Blunt ${ }^{\circledR}$ PCR Cloning Kit (Invitrogen), sequenced, and annotated to confirm its homology to $D A M$ genes from other plant species (data not shown). The alignment of protein sequences of this initial partial DAM fragment, pPpMADS13\#2, revealed high amino acid conservation among the japanese pear and Prunus DAM proteins (data not shown). The nucleotide sequences of the $p P p M A D S 13 \# 2$ showed high conservation to the corresponding regions of the later-identified full-length PpMADS13-1 (86\%) and PpMADS13-2 (99\%), but neither was identical to both. This conserved initial PCR fragment, $p P$ PADS13\#2, was chosen in the Southern blotting experiment to estimate the copy number of the $D A M$ genes in the pear genome.

Southern blotting Analysis to Determine the DISTRIBUTION OF THE DAM GENES IN THE GENOME OF JAPANESE PEAR. Southern blotting analysis was carried out with genomic DNA of 'Kosui'. Hybridization under moderate stringency conditions revealed one to two major bands and a number of weak fragments, suggesting that several copies of the pPpMADS13\#2 may be present in the pear genome (Fig. 1A).

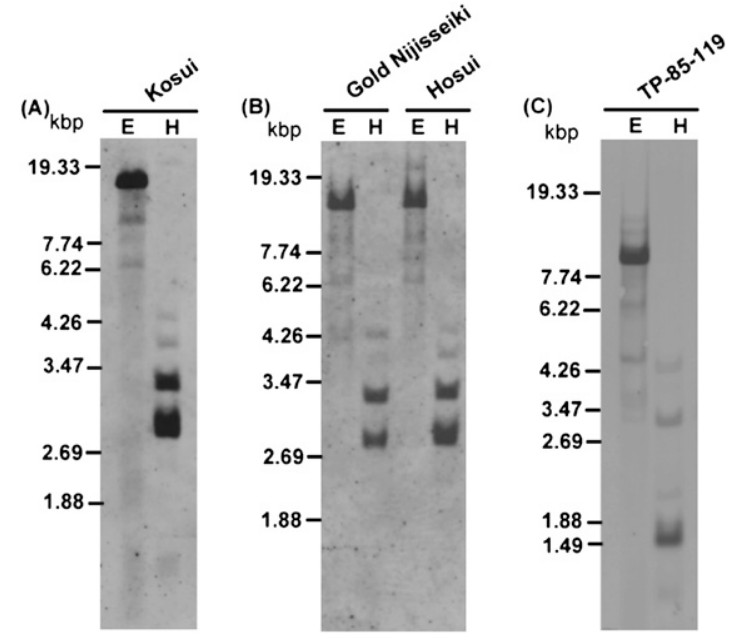

Fig. 1. Southern blotting analysis showing distribution of the $D A M$ genes in the pear genome: japanese pear ['Kosui' (A), 'Gold Nijisseiki' and 'Hosui' (B)] and taiwanese pear [TP-85-119 (C)]. Ten micrograms of genomic DNA was digested with EcoRI (E) and HindIII (H). The DIG-labeled partial DAM fragment, $p P p M A D S 13 \# 2$ (609 bp) was used as a hybridization probe. Positions of molecular size markers are shown at the left.

Southern blotting analysis involving the genomic DNA of two other japanese pear cultivars (Gold Nijisseiki and Hosui) digested with the same enzymes and probe also yielded the same banding patterns as 'Kosui' (Fig. 1B). In addition, Southern blotting analysis of the taiwanese pear (TP-85-119) showed similar banding patterns, which indicated that the DAM genes are also present in the TP-85-119 genome. However, differences in the lower bands between TP-85-119 and the japanese pear type were observed under similar hybridization conditions (Fig. 1C).

Cloning OF FUll-Length CDNAS OF PEAR DAM GENES AND SEQUENCE ANALYSIS. To isolate full-length cDNA of japanese pear $D A M$ genes from 'Kosui', 3'- and 5'-RACE was performed using the corresponding gene-specific primers designed based on the 609-bp partial fragment ( $p$ P $M A D S 13 \# 2)$. The first and second 5'-RACE produced fragments of 471 and $459 \mathrm{bp}$, respectively, while the $3^{\prime}$-RACE produced a 411-bp product. The partial 609-bp RT-PCR fragment and the RACE fragments were aligned to obtain a 1191-bp contig containing a 239-bp 5'-noncoding region, a 705-bp open reading frame (ORF), and a 247-bp $3^{\prime}$-noncoding region. Forward and reverse primers were designed based on this contig for the isolation of the full-length cDNAs. Sequencing of 20 randomly selected clones produced two groups of clones that we designated as PpMADS13-1 and PpMADS13-2, with each group of clones possessing $100 \%$ nucleotide sequence similarity. The ORF of PpMADS13-1 encodes a 227-amino acid polypeptide with a predicted molecular mass of $25.1 \mathrm{kDa}$ and an isoelectric point $(p I)$ of 5.5, while the PpMADS13-2 ORF encodes a 234-amino acid polypeptide with a $26.4 \mathrm{kDa}$ predicted molecular mass and a $p I$ of 8.4 . The alignment of the complete PpMADS13-1 and PpMADS13-2 protein sequences revealed high amino acid conservation among pear and Prunus DAM proteins, including the LEDDCSD motif (Jiménez et al., 2009) at amino acid positions 233 to 239 in the C-terminal region (Fig. 2). Moreover, no serine-aspartate change, which exists in peach DAM1 and DAM3, at this potential positively selected site (amino acid 
PaMADS1 MVKMMRKKIK IKKIDCLPAR QVTFSKRRRG IFKKAAELSV LCESKVAVVI FSATGKLFDY SSSSTKDVIE RYKAHTNGVE KSDEPSVELQ Ppdam3 MVKMMRKKIK IKKIDYLPAR QVTFSKRRRG IFKKAAELSV LCESKVAVVI FSATGKLFDY SSSS IKDVIE RYKAHTNGVE KSDKPSVELQ Ppdam1 - MKMTREKIK IKKIDNLPAR QVTFSKRRRG IFKKAAELSV LCESEVAVVI FSATGKLFDY SSSSMKDVIE RYQEH INGAE KFDEPS IELQ Ppdam6 -- MMREKIK IKKIDYLPAR QVTFSKRRRG LFKKAAELSV LCESEVAVI I FSATDKLFDY SSSSTEDVIE RYKAHTNDLE KSNKQFLELQ Pmdam6 MVKMMREKIK IKKIDYLPAR QVTFSKRRRG LFKKAAELSV LCESEVAVVI FSATDKLFHY SSSSTENVIE RYKAHTGGVE KSDKQFLELQ Ppdam4 MVKMMREKIK IKKIDYLPAR QVTFSKRRRG IFKKAAELSV LCESEVAVVI FSATGKLFDY SSSS IKDVIE RYEVRTNGVE KSDEQSLELQ Ppdam5 - - MMRNKIK IKKIDYLPAR QVTFSKRRRG LFKKAAELSV LCESEVAVVI FSATGKLFDY SSSSTKDVIE RYNADINGVE KLNNQEIELQ Ppdam2 MVKTMRKKIK IKKIDYLPAR QVTFSKRRRG IFKKAEELSV LCESEVAVVI FSATGKLFDY SSSSTKDVVE RYQAHTNGVE KSDEPSVELQ PpMADS13-1 -.--- MKIK IRKIDYLPAR QVTFSKRRRG IFKKAGELSI LCESEVAVI I FSQTGKLFDF SSSSTKDVIA RYNSHVGGEK SDQPTLHQLL PpMADS13-2 MVKRMNEKIK IRRIDYLPAR QVTFSKRRRG IFKKAEELSI LCESEVAVI I FSQTGKLFDY SSSSTKDVIA RYKLHTGGEK SDQITLHQLQ

\begin{tabular}{|c|c|c|c|c|c|c|c|}
\hline & 100 & 110 & 120 & 130 & 140 & 170 & 180 \\
\hline PaMADS1 & LENENHIGLS & KELEEKSHQL & RQMKAEDLEE & LNFDELQKLE & QLVDASLGRV I ETKEELRMS & EIMALERKGA ELVEANN--- & QLRQTMVMLS \\
\hline Ppdam3 & LENENQIGLS & KELKEKSHQL & RQMKAEDLEE & LNFDELQKLE & QLVDASLGRV I ETKEELRMS & EIMALERKGA ELVEANN--- & TM -MLS \\
\hline Ppdam1 & PEKENHIRLS & KELEEKSRQL & RQMKGEDLEE & LNFDELQKLE & QLVDASLGRV I ETKDELI MS & EIMALKRKRA ELVEANK--- & QLRQRASNYH \\
\hline Ppdam6 & LENENHIKLS & KELEEKSRQL & RQMKGEDLQG & LNMDELLKLE & LGRV I ETKEELI MS & EIMALEKKGA ELVE & AMLS \\
\hline Pmdam6 & LENENHIKLS & KELEEKSRQL & MKGEDLEG & LNLDELLKLE & QLVEASLGRV I ETKEELI MS & EIMALEKKGA EL & VMLS \\
\hline Ppdam4 & LENENHTKLS & TELEEKNRQL & LEE & LDLDELLKLE & LVRV METKEELIMS & LEKKGT EL & VMLS \\
\hline Ppdam5 & LENENHIKLS & KELEEKSRQL & RQMKGEDLEG & LNLDELLKLE & QLVEASLGRV METKEELIKS & EIMALERKGT ELVEANN--- & VMLS \\
\hline Ppdam2 & LEIENHIRLT & KELEEKSCQL & RQIKGEDLEE & LNFDELQKLE & QLVDASLGRV IETEEELIMS & EIMALERKGA EL & VMLS \\
\hline PpMADS & 1 LEKENNIRLS & KELEDKSCKL & RQMKGVDLED & LDLGELQKLE & KLVEASLGRV IQTKEEKITS & EKKGA EI & IVMLP \\
\hline PpMADS13- & 2 SEKENTI RLS & KELEDKTRKL & RQMKGEDLQD & LDLYQLNKLE & KLVEASVGRV IKTKEKKIMS & EIMALTNKGA ELIEANN--- & QLKQRLVMLS \\
\hline
\end{tabular}

\begin{tabular}{|c|c|c|c|c|c|c|c|c|c|c|}
\hline & 190 & 200 & 210 & 220 & 230 & & & 40 & 250 & 260 \\
\hline PaMADS1 & -----GGNTG & PELMEPERLN & NYTGGGGEEE & GMSTESAIST & TCNSAHS--- & -- & LGDDSD- & $\mathrm{N}$ & VTLSLKLGLP & \\
\hline Ppdam3 & -----GGNTG & PTLMEPERLS & NNIGGGGEEE & GMSSESAIST & TCNSALSLSP & $-\mathrm{S}$ & LGDDSDD & - & VTLSLKLGLS & \\
\hline Ppdam1 & NHMLSRGNIG & PALMEPERLN & NNIGGGGEEE & GMSSESATST & TCNSAPSL -- & $-\mathrm{S}$ & LEDDSDD & - & VTLSLKLGLP & \\
\hline Ppdam6 & -----GGNTG & PAFVEPETLI & TNVGGGGEED & GMSSESAIIA & TSTSCNSAHS & LS & LEDDCSD & - & VTLSLKLGLP & \\
\hline Pmdam6 & -----GGNTG & PAFVEPETLI & TNVGGGGGED & DMSSESAVIA & TSTSCNSAFS & $\mathrm{LS}$ & LEDDCSD & - & VTLSLKLGLP & ----. \\
\hline Ppdam4 & -----KRNTG & PALMEP---- & ---------- & ------SESA & TSTSCNSALS & $\mathrm{LS}$ & LEDDCSD & $\mathrm{D}$ & VVLSLKLGLT & VRAGRRPMCL \\
\hline Ppdam5 & -----GGNTG & PALMDPERLN & NNIEGGGEEE & GMSAESAIST & TCNSAVS--- & $\mathrm{LS}$ & LEDDSSD & $E$ & VTLSLKLGR- & --- \\
\hline Ppdam2 & -----RGNIG & PAPTEPERFV & NNIGGGGEEG & - MSSESATNA & TISSCSSGPS & $\mathrm{LS}$ & LEDDCSD & - & VTLALKLGLP & \\
\hline PpMADS13-1 & G-----GDSG & PEAI LELENL & NNIGEG---- & SVTSESATNV & TTFSNSS--- & $\mathrm{LS}$ & LEDDCSD & - & -TLSLKLGLP & \\
\hline PpMADS13-2 & AR----GDIE & PAAIMELENL & NNVGEE---- & GMTSESATNV & TACSSSA & $\mathrm{LS}$ & LEDDCSD & - & -ILSLKLGLP & ------------ \\
\hline
\end{tabular}

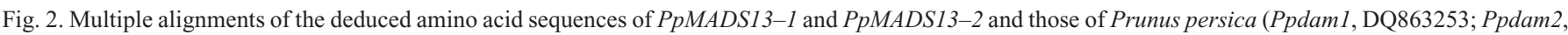
DQ863255; Ppdam3, DQ863256; Ppdam4, DQ863250; Ppdam5, DQ863251; and Ppdam6, DQ863252), Prunus mume (Pmdam6, AB437345), and Prunus avium (PaMADS1, EU196363). Sequence comparison was obtained using ClustalW and included M, I, K, and C domains. The LEDDCSD motif in the C-terminal region is boxed.

position 238) has occurred in the PpMADS13-1 and PpMADS13-2 (Jiménez et al., 2009). The similarity score for pairwise comparison of the amino acid sequences between PpMADS13-1 and PpMADS13-2 was 81.0\%.

Sequence analysis by a tBLASTx similarity search against the NCBI nonredundant database revealed that PpMADS13-1 and $P p M A D S 13-2$ are highly similar to known $D A M$ genes identified in several other species (Table 1), which is consistent with the partial fragment used as hybridization probe for Southern analysis (data not shown). Alignment of the predicted amino acid sequences from these sequences showed that PpMADS13-1 and PpMADS13-2 contained the well-conserved MIKC-motifs characteristic of DAMS (Fig. 2). The M-domain, which is important in homeotic regulation, is about 60 amino acids long. The variable I-domain, consisting of about 30 amino acids, links the M- and K-domains. The K-domain is about 70 amino acids long and is linked to the most variable and functionally important C-terminal domain. A phylogenetic tree constructed based on the complete amino acid sequences (M, I, $\mathrm{K}$, and C domains) of PpMADS13-1 and PpMADS13-2 and other $17 D A M$ genes (Fig. 3) revealed that the pear $D A M$ genes are more closely related to those of Prunus species. However, the pear $D A M$ genes formed an independent subclade.

SEasonal CHanges IN THE ENDOdORManCY TRANSITION PHASES AND EXPRESSION ANAlysis OF PPMADS13-1 AND PrMADS13-2 GENES. To examine the changes in lateral leaf bud gene expression during the endodormancy transition phases, it was necessary to define the dormancy status of the lateral leaf buds during the endodormancy transitional phases. The seasonal changes in the endodormancy transition phases assessed in the lateral leaf buds from early Sept. 2008 to midFeb. 2009 are shown in Fig. 4A. In early September, the sprouting percentage in 'Kosui' was $8 \%$ and declined to $0 \%$ throughout the 21 Oct., 12 Nov., and 9 Dec. sampling dates when a sharp increase occurred, reaching 56\%,79\%, and $93 \%$ on 24 Dec., 8 Jan., and 12 Feb., respectively (Fig. 4A). These results suggest that 'Kosui' leaf buds were released from endodormancy about mid-December and progressed to ecodormant stage by late December. The less dormant TP-85-119 had a sprouting ratio of $82 \%$ (12 Nov.) and 95\% (9 Dec.) when that of the endodormant 'Kosui' was $0 \%$.

The specific gene expression patterns of the PpMADS13-1 and $P$ PMADS13-2 genes were investigated in the lateral leaf buds of 'Kosui' through seasonal dormancy transitions. The quantitative real-time PCR showed that the PpMADS13-1 and PpMADS13-2 genes were differentially expressed in relation to the endodormancy status in the lateral leaf buds of 'Kosui' (Fig. 4, B and C). The expression level of both DAM genes fluctuated similarly during the period from early September to midFebruary and showed some association with endodormancy phase transitions. However, changes in gene expression levels were relatively slower than changes in endodormancy status, especially toward endodormancy release. Both genes had a low expression level by 9 Sept., gradually increased to a peak 


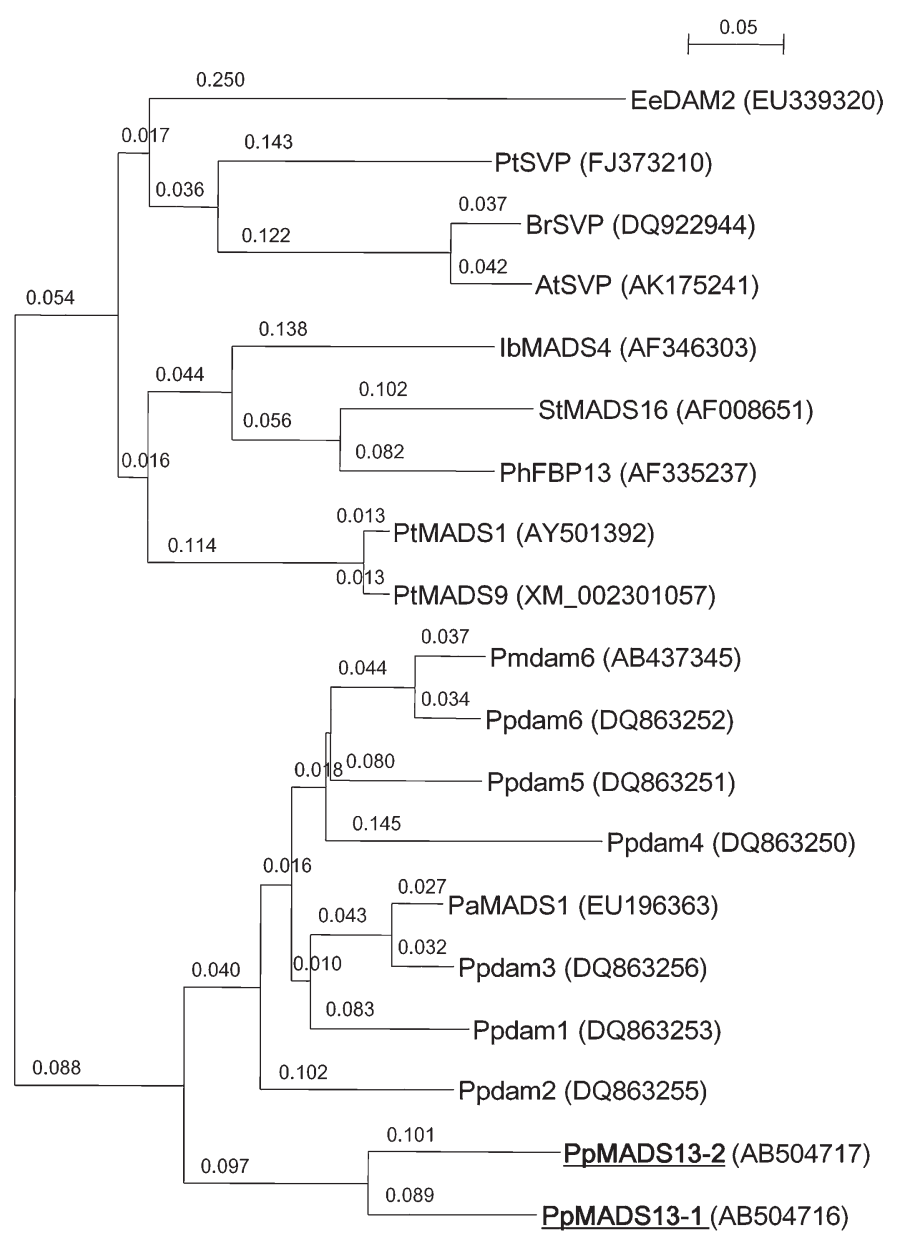

Fig. 3. A phylogenetic tree constructed based on the complete amino acid sequences of the 'Kosui' japanese pear PpMADS13-1 and PpMADS13-2 (underlined), Prunus persica (DQ863253, DQ863255, DQ863256, DQ863250, DQ863251, and DQ863252), Prunus mume (AB437345), Prunus avium (EU196363), Populus trichocarpa (XM_002301057), Populus tomentosa (AY501392), Petunia $\times$ hybrida (AF335237), Solanum tuberosum (AF008651), Ipomoea batatas (AF346303), Arabidopsis thaliana (AK175241), Brassica rapa subsp. campestris (DQ922944), Poncirus trifoliata (FJ373210), and Euphorbia esula (EU339320). The values indicate the branch length.

expression level concomitant with endodormancy by 9 Dec., and then gradually decreased to a low level on $12 \mathrm{Feb}$. The PpMADS13-2 DAM gene was generally more highly expressed than PpMADS13-1 (Fig. 4, B and C). The expression of PpMADS13-1 was low by 9 Sept., when the sprouting ratio was $8 \%$, increased sharply to a 33 -fold maximal level at the peak of endodormancy by $9 \mathrm{Dec}$. when the sprouting ratio was $0 \%$, and then gradually decreased with increasing sprouting ratio (endodormancy release) to an $\approx 8$-fold lower level by 12 Feb. (when the sprouting ratio was $93 \%$ ), relative to 9 Dec. (Fig. 4B). It is worth noting that the $D A M$ genes not only showed a decreased expression with endodormancy release, but also a build up with the installation of endodormancy. The expression level of PpMADS13-1 in the less dormant TP-85-119 was very significantly lower (nearly zero level) on 12 Nov. (82\% sprouting ratio) and $9 \mathrm{Dec}$ ( $95 \%$ sprouting ratio), relative to the endodormant 'Kosui' (Fig. 4B). Similarly, the expression of PpMADS13-2 in 'Kosui' was low by 9 Sept., when the sprouting ratio was $8 \%$, increased sharply to a 5 -fold maximal
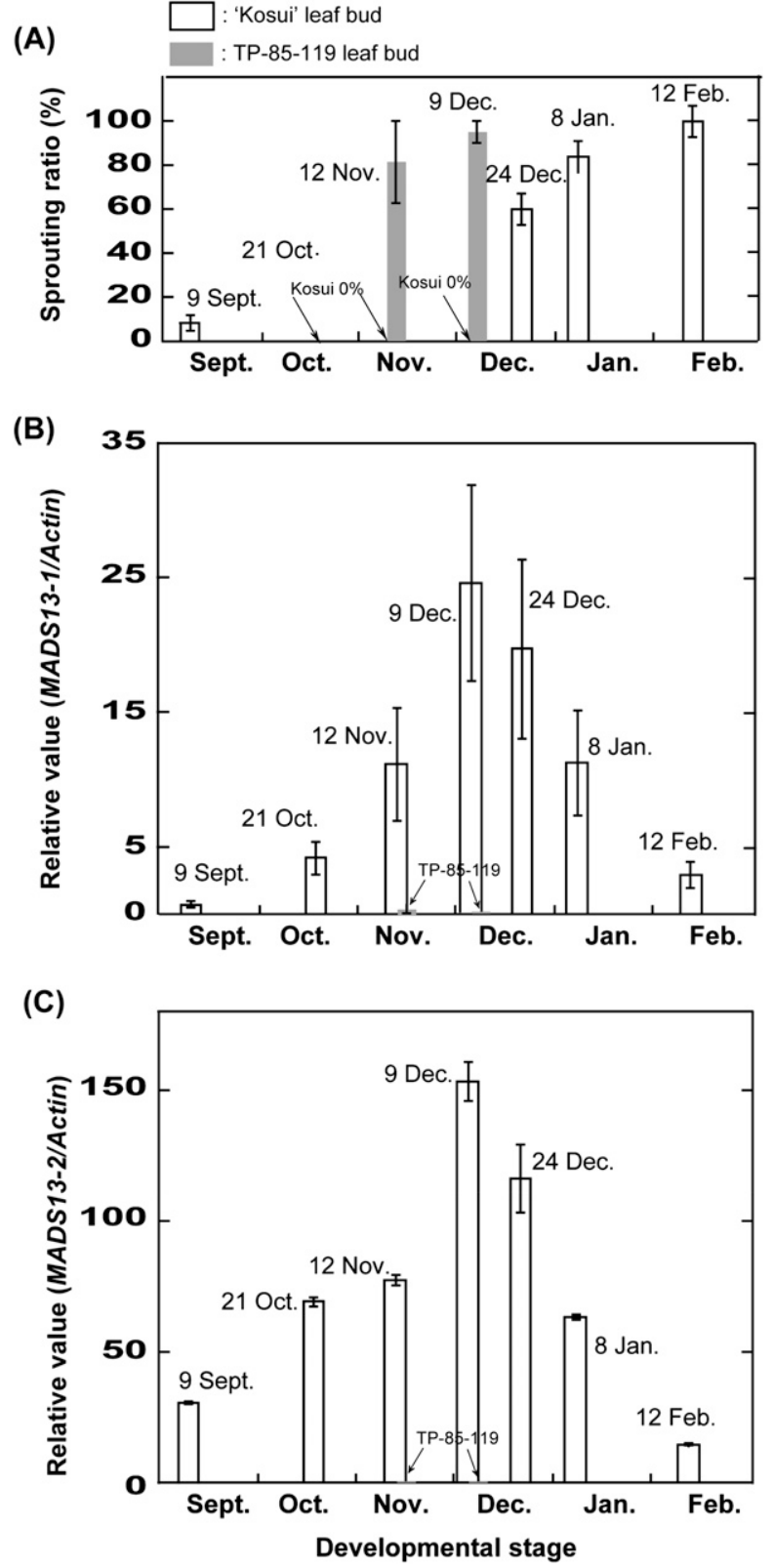

Fig. 4. Sprouting ratio (in percentage) (A), relative expression levels of PpMADS13-1 (B), and relative expression levels of PpMADS13-2 (C) in the lateral leaf bud during the endodormancy transition phases in 'Kosui' japanese pear. Data of the less dormant taiwanese pear, TP-85-119 in (A-C) is shown in gray.

level at the peak of endodormancy by 9 Dec. (when the sprouting ratio was $0 \%$ ), and then gradually decreased with increasing sprouting ratio (endodormancy release) to a 10 -fold lower level by $12 \mathrm{Feb}$. (when the sprouting ratio was $93 \%$ ), relative to 9 Dec. (Fig. 4C). The expression level of PpMADS13-2 in the less dormant TP-85-119 was also very significantly lower (nearly zero level), as with the expression of PpMADS13-1 (Fig. 4B), on 12 Nov. (82\% sprouting ratio) and 9 Dec. (95\% sprouting ratio) relative to the endodormant 'Kosui' (Fig. 4C). Unlike PpMADS13-2, the expression level of PpMADS13-1 on 12 Feb. was relatively higher (4-fold) than on 9 Sept. (when the sprouting ratio was just $8 \%$ ), suggesting the possible role of environmental factors in the level of 
expression of these genes in 'Kosui'. Overall, the expression patterns of PpMADS13-1 and PpMADS13-2 genes in lateral leaf buds showed a gradual down-regulation of the genes upon endodormancy release.

Methylation status of PpMAdS13-1 and PpMADS13-2 DURING THE ENDODORMANCY TRANSITION PHASES. Southern blotting analysis using genomic DNA from the lateral vegetative buds from 'Kosui' during the endodormancy transition phases (from mid-October to mid-February) and specific PpMADS131 and $P p M A D S 13-2$ gene probes showed differences in the fragments detected by HpaII and MspI, which reflects methylation at the $\mathrm{CpG}$ sites (Fig. 5, A and B). However, DNA methylation in both loci may not be associated with the endodormancy phenomenon, as identical banding patterns were produced in the CCGG allelic sites that were not concomitant with the endodormancy transition phases as shown in Fig. 4A. Several common bands were observed between PpMADS13-1 and PpMADS13-2, suggesting high similarity in the genomic organization between these genes.

\section{Discussion}

The six MIKC-type $D A M$ genes described in peach (Bielenberg et al., 2004, 2008; Wang et al., 2002) are candidate genes for the regulation of growth cessation and terminal bud formation in peach. The $D A M$ genes are down-regulated concomitant with endodormancy release and are not detected in the evergrowing mutant (Bielenberg et al., 2008). Since their discovery, interest has been growing in the investigation of these candidate genes in other perennial species, including raspberry, japanese apricot, and leafy spurge (Horvath et al.,
(A)

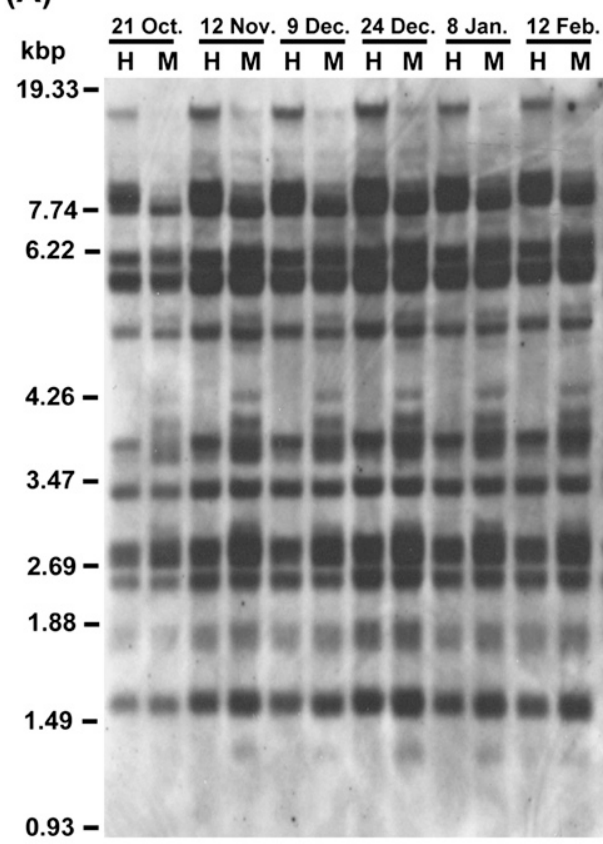

(B)

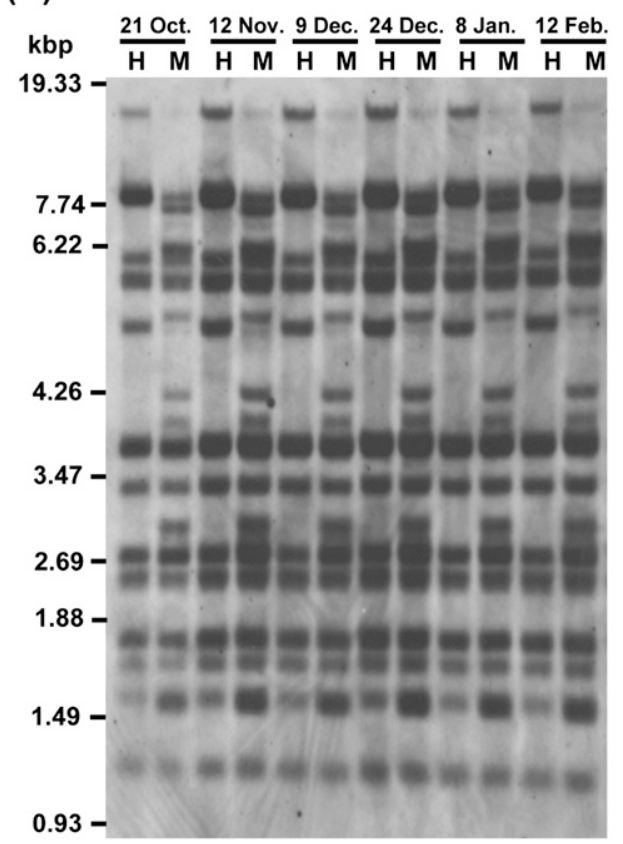

Fig. 5. Methylation analysis of PpMADS13-1 (A) and PpMADS13-2 (B) locus. Lateral leaf bud was collected from 'Kosui' japanese pear at six different seasonal dormancy transition stages from 2008 (21 Oct., 12 Nov., 9 Dec., and 24 Dec.) and 2009 (8 Jan. and 12 Feb.). Genomic DNA (10 $\mu \mathrm{g}$ ) was digested with the methylationsensitive isoschizomer pair HpaII (H) and MspI (M). Specific DIG-labeled coding sequences for PpMADS13-1, (A, $674 \mathrm{bp}$ ) and PpMADS13-2, (B, $701 \mathrm{bp}$ ) were used as hybridization probes. $\mathrm{H}=$ HpaII, $\mathrm{M}=$ MspI. Positions of molecular size markers are shown at left.
2008; Mazzitelli et al., 2007; Yamane et al., 2008). In this study, we isolated two full-length $D A M$ genes in 'Kosui' japanese pear, PpMADS13-1, and P PMADS13-2, investigated their expression, as well as their methylation status during the endodormancy transitional phases in japanese pear.

Southern blotting analysis with EcoRI- and HindIII-digested DNA of three japanese cultivars using a partial DAM gene sequence ( $p P p M A D S 13 \# 2)$ as a probe was performed. It should be noted that the probe can detect at least PpMADS13-1 and PpMADS13-2 genomic regions under moderate stringency because $p P p M A D S 13 \# 2$ share high sequence similarities between PPMADS13-1 and PpMADS13-2. The analysis yielded one or two major bands and a number of weak bands, suggesting that several copies of the $p P p M A D S 13 \# 2$ may be present in the pear genome. Southern blotting analysis of the taiwanese pear (TP-85-119) performed under different electrophoresis conditions also showed similar banding patterns, albeit the observed differences in the lower bands, indicating that taiwanese pear also possesses similar genomic structure for pPpMADS13\#2. The major hybridizing bands detected in this study were of greater intensity, which may result from being the sequence of highest similarity to the hybridizing probe or, more likely, the possibility of potentially two or more overlapping bands of this gene family. However, these possibilities remain to be clarified. In addition, because only $p P p M A D S 13 \# 2$ was tested in this study, further study is required to confirm the presence of other $D A M$ genes in the pear genome like peach (Bielenberg et al., 2004). Jiménez et al. (2009) indicated that the members of the DAM genes of the SVP/StMADS11-like type in peach (six) follow the trend of increased number as in other perennial species such as poplar [Populus spp. (eight)] and grape [Vitis vinifera (five)] relative to the decreased number reported in other annual species, including A. thaliana (two), tomato [Solanum lycopersicum (two)], and rice [Oryza sativa (three)].

Two full-length $D A M$ genes cloned from japanese pear have high sequence conservation and showed a high degree of identity with $D A M$ genes from other plant species at the amino acid level. A conserved motif containing a potential positively selected site (LEDDCSD) of the C-terminal region reported among the peach MIKC-type MADS-box genes was also identified in this study. No serine(S)-aspartate(D) change occurred at this site of the PpMADS13-1 and PpMADS13-2 genes, unlike in peach $D A M 1$ and $D A M 3$. This position had been reported to have a posterior possibility of $>0.95$ and was likely to be functionally significant (Jiménez et al., 2009). As yet, we do not know the number of candidates of this gene family in japanese pear, and their complete identification will confirm whether this single amino 
Table 1. Identity of 'Kosui' japanese pear MADS 13-1 and MADS13-2 from a tBlastx similarity search on 16 June 2009 against the NCBI nonredundant (nr) database of 10 top-hit dam genes.

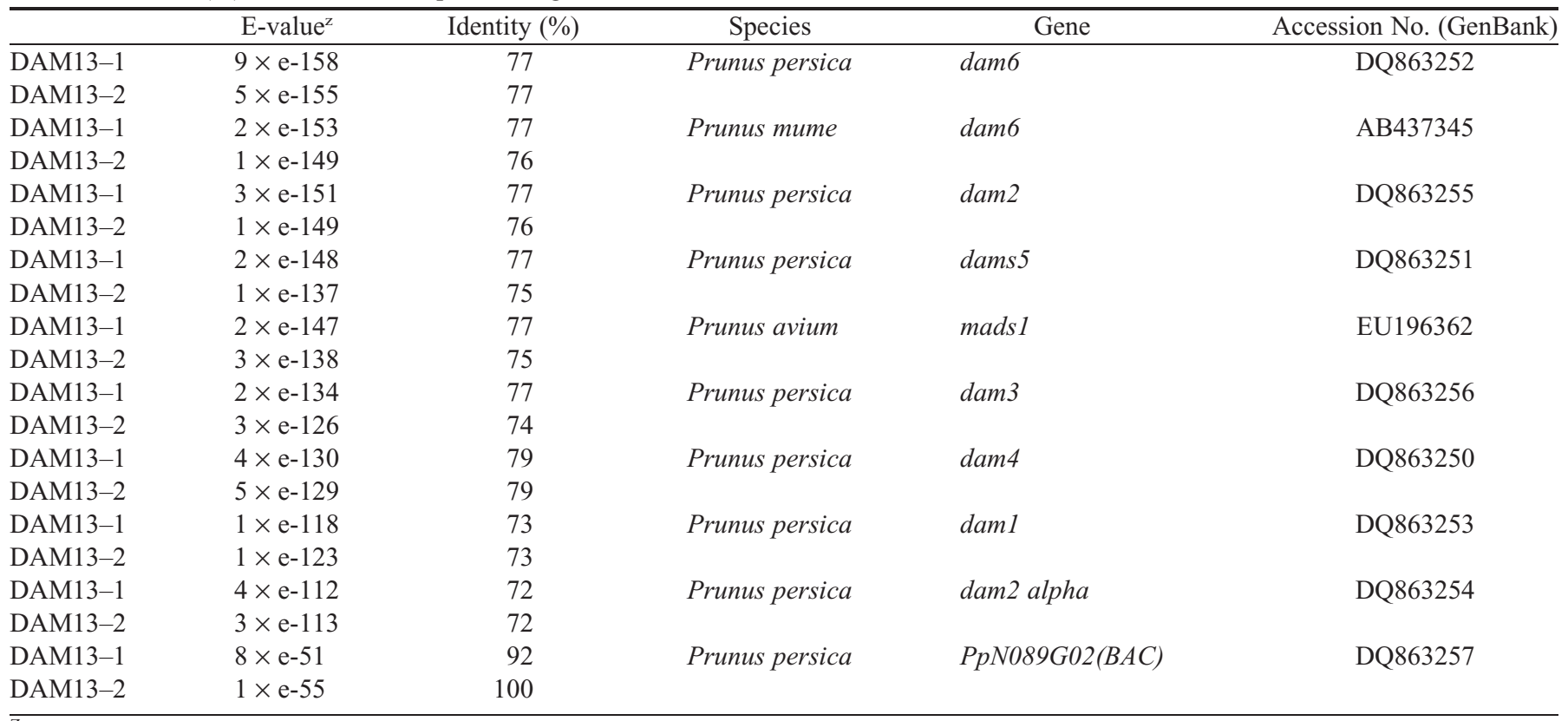

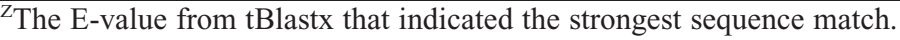

acid residue is also under significant positive selection among the japanese pear DAMS. These results may suggest that the evolutionary divergence in the species does not result in high rates of nucleotide substitutions among the $D A M$ genes of japanese pear, peach, and japanese apricot. Relationship analysis based on the phylogenetic analysis constructed using the complete amino acid sequences demonstrated that PpMADS13-1 and PpMADS13-2 were more closely related to Prunus DAM genes than those of other taxa. However, PpMADS13-1 and PpMADS13-2 formed an independent subclade from the Prunus DAM genes, suggesting uniqueness based on taxonomic distance. Based on the differences in amino acid sequences, it seems that PpMADS13-1 and PpMADS13-2 may likely be derived from different loci, rather than being alleles of the same locus. However, the possibility of an allelic relationship cannot be completely ruled out. In future studies, the possibility of adding new pear $D A M$ homologs and phylogenetic analysis will clarify the relationships in the $D A M$ genes of Pyrus and other members of this gene family. Indeed, work is currently underway to annotate all of the gene candidates of this gene family using a microarray approach to identify other members of this gene cluster in japanese pear.

This study has identified important gene candidates of seasonal endodormancy regulation in japanese pear. The pattern of expression in the two $D A M$ genes fluctuated similarly with the seasonal endodormancy phase transitions (induction and release) in 'Kosui' lateral leaf buds, although PpMADS13-2 was more highly expressed than PpMADS13-1. The peak expression in both $D A M$ genes occurred on 9 Dec., which coincided with the probable transition phase of deep endodormancy in 'Kosui' before the gradual onset of endodormancy release. Similarly, the expression of these two DAM genes in the less dormant TP-85119 PI was consistently quite low. Although we do not yet know the sequence information of these $D A M$ genes in taiwanese pear, our real-time PCR results (based on the analysis of the dissociation curve) indicated reliable amplification by the primers. Therefore, the quite low expression of these genes in the TP-85-119 is consistent with the down-regulation that is associated with the near loss of endodormancy in this taiwanese pear. These results on the seasonal expression of the two DAM genes in pear are consistent with the results reported in other species, including Prunus species, where the DAM genes were down-regulated with endodormancy release (Bielenberg et al., 2008; Li et al., 2009; Yamane et al., 2008).

To test the hypothesis of epigenetic gene regulation of endodormancy involving genome methylation, we investigated the methylation patterns in the genomic region underlying PpMADS13-1 and PpMADS13-2 genes using genomic DNA of 'Kosui' in the course of the endodormancy transition phases (late October to early February). Our results revealed that although DNA methylation occurred in these genes, it is not concomitant with the changes in the gene expression that are associated with endodormancy status, as detected by the isoschizomer pair HpaII/MspI.

Collectively, our results are consistent with the hypothesis of a down-regulated pattern of expression for the DAM genes. Our finding in japanese pear is consistent with reports in other deciduous tree species where a gradual down-regulation of the $D A M$ gene was correlated with endodormancy release (Horvath et al., 2008; Mazzitelli et al., 2007; Yamane et al., 2008). As has been stated earlier (Bielenberg et al., 2008), a disruption of gene function of these candidate genes, leading to a loss of expression, may be responsible for the nondormant condition in the evergrowing mutant types carrying a deletion of the DAM genes. As highlighted by Yamane et al. (2008), members of this gene family might function as internal suppressor genes to delay or inhibit bud growth under prevailing favorable conditions for bud burst when none of the external inhibitory effects (e.g., apical dominance or unsuitable environmental conditions) exist. The differential expression patterns of PpMADS13-1 and PpMADS13-2 genes at the last stage of sampling (12 Feb.), relative to 9 Sept. (the stage of progression into endodormancy) 
suggest a possible effect of different dormancy-inducing environmental cues (such as low temperatures in Pyrus) on their seasonal expression. Recent findings have also indicated that $D A M$ genes may not only be required for endodormancy induction, but that their induction may be signaled by environmental cues known to regulate endodormancy, such as cold temperatures or short days (Horvath et al., 2008; Li et al., 2009). It is worth noting the fact that although Pyrus and Prunus are species from the same family, fundamentally different environmental cues trigger their growth cessation, bud formation, and endodormancy induction (Horvath et al., 2008; Li et al., 2009). While short photoperiods are one of the important signals to trigger endodormancy in peach, short photoperiods have no effect in japanese pear, but low temperatures are solely responsible for the induction of bud formation and endodormancy (Heide and Prestrud, 2005). Further studies involving the isolation and functional analysis of the CONSTANS gene $(C O)$ will be necessary for a clear-cut explanation of this difference between pear and peach. Moreover, a FLOWERING LOCUS T $(F T)$-like gene that was hypothesized to interact with DAM proteins to regulate dormancy transitions was shown to be reciprocally regulated with the expression of $D A M$ genes in leafy spurge (Horvath et al., 2008). Therefore, investigating the expression of these candidate $D A M$ and $F T$-like genes in pear under low temperatures will also be worthwhile. We are currently isolating TERMINAL FLOWER (TFL)- and FT-like genes to confirm this hypothesis in japanese pear.

\section{Literature Cited}

Arora, R., L.J. Rowland, and K. Tanino. 2003. Induction and release of bud dormancy in woody perennials: A science comes of age. HortScience 38:911-921.

Bielenberg, D.G., Y. Wang, S. Fan, G.L. Reighard, R. Scorza, and A.G. Abbott. 2004. A deletion affecting several gene candidates is present in the evergrowing peach mutant. J. Hered. 95:436-444.

Bielenberg, D.G., Y. Wang, Z. Li, T. Zhebentyayeva, S. Fan, G.L. Reighard, R. Scorza, and A.G. Abbott. 2008. Sequencing and annotation of the evergrowing locus in peach [Prunus persica (L.) Batsch] reveals a cluster of six MADS-box transcription factors as candidate genes for regulation of terminal bud formation. Tree Genet. Genomes 4:495-507.

Crabbe, J. and P. Barnola. 1996. A new conceptual approach to bud dormancy in woody plants, p. 83-113. In: G.A. Lang (ed.). Plant dormancy: Physiology, biochemistry, and molecular biology. CAB International, Wallingford, UK.

de Castro, E., C.J.A. Sigrist, A. Gattiker, V. Bulliard, S. Petra, P.S. Langendijk-Genevaux, E. Gasteiger, A. Bairoch, and N. Hulo. 2006. ScanProsite: Detection of PROSITE signature matches and ProRuleassociated functional and structural residues in proteins. Nucleic Acids Res. 34:W362-W365.

Dennis, E.S. and W.J. Peacock. 2007. Epigenetic regulation of flowering. Curr. Opin. Plant Biol. 10:520-527.

Faust, M., A. Erez, L.J. Rowland, S.Y. Wang, and H.A. Norman. 1997. Bud dormancy in perennial fruit trees: Physiological basis for dormancy induction, maintenance, and release. HortScience 32:623-629.

Finnegan, E.J., W.J. Peacock, and E.S. Dennis. 2000. DNA methylation, a key regulator of plant development and other processes. Curr. Opin. Genet. Dev. 10:217-223.

Genger, R.K., W.J. Peacock, E.S. Dennis, and E.J. Finnegan. 2003. Opposing effect of reduced DNA methylation on flowering time in Arabidopsis thaliana. Planta 216:461-466.

Heide, O.M. and A.K. Prestrud. 2005. Low temperature, but not photoperiod, controls growth cessation and dormancy induction and release in apple and pear. Tree Physiol. 25:109-114.
Henderson, I.R. and C. Dean. 2004. Control of arabidopsis flowering: The chill before the bloom. Development 131:3829-3838.

Horvath, D.P., J.V. Anderson, W.S. Chao, and M.E. Foley. 2003. Knowing when to grow: Signals regulating bud dormancy. Trends Plant Sci. 8:534-540.

Horvath, D.P., W.S. Chao, J.C. Suttle, J. Thimmapuram, and J.V. Anderson. 2008. Transcriptome analysis identifies novel responses and potential regulatory genes involved in seasonal dormancy transitions of leafy spurge (Euphorbia esula L.). BMC Genomics 9:536.

Jiménez, S., A.L. Lawton-Rauh, G.L. Reighard, A.G. Abbott, and D.G. Bielenberg. 2009. Phylogenetic analysis and molecular evolution of the dormancy associated MADS-box genes from peach. BMC Plant Biol. 9:81.

Keilin, T., X. Pang, J. Venkateswari, T. Halaly, O. Crane, A. Keren, A. Ogrodovitch, R. Ophir, R. Volpin, D. Galbraith, and E. Or. 2007. Digital expression profiling of a grape-bud EST collection leads to new insight into molecular events during grape-bud dormancy release. Plant Sci. 173:446-457.

Lang, G.A. 1987. Dormancy: A new universal terminology. HortScience 22:817-820.

Li, Z., G.L. Reighard, A.G. Abbott, and D.G. Bielenberg. 2009. Dormancy-associated MADS genes from the EVG locus of peach [Prunus persica (L.) Batsch] have distinct seasonal and photoperiodic expression patterns. J. Expt. Bot. 60:3521-3530.

Mazzitelli, L., R.D. Hancock, S. Haupt, P.G. Walker, S.D.A. Pont, J. McNicol, L. Cardle, J. Morris, R. Viola, R. Brennan, P.E. Hedley, and M.A. Taylor. 2007. Coordinated gene expression during phases of dormancy release in raspberry (Rubus ideaeus L.) buds. J. Expt. Bot. 58:1035-1045.

Rodriguez, J., W.B. Sherma, R. Scorza, M. Wisniewski, and W.R. Okie. 1994. Evergreen peach, its inheritance and dormant behavior. J. Amer. Soc. Hort. Sci. 119:789-792.

Rohde, A. and R.P. Bhalerao. 2007. Plant dormancy in the perennial context. Trends Plant Sci. 12:217-223.

Rozen, S. and H.J. Skaletsky. 2000. Primer 3 on the WWW for general users and for biological programs, p. 365-386. In: S. Krawetz, and S. Misener (eds.). Bioinformatics methods and protocols: Methods in molecular biology. Humana Press, Totowa, NJ.

Sugiura, T., H. Kuroda, and H. Sugiura. 2007. Influence of the current state of global warming on fruit tree growth in Japan. Hort. Res. (Japan) 6:257-263 (In Japanese with English summary).

Thomas, M.R., S. Matsumoto, P. Cain, and N.S. Scott. 1993. Repetitive DNA of grapevine: Classes present and sequences suitable for cultivar identification. Theor. Appl. Genet. 86:173-180.

Thompson, J.D., D.G. Higgins, and T.J. Gibson. 1994. CLUSTAL W: Improving the sensitivity of progressive multiple sequence alignment through sequence weighting, position-specific gap penalties and weight matrix choice. Nucleic Acids Res. 22:4673-4680.

Thompson, M.M., D.C. Smith, and J.E. Burgess. 1985. Non-dormant mutants in the temperate tree species, Corylus avellana L. Theor. Appl. Genet. 70:687-692.

Wan, C. and T.A. Wilkins. 1994. A modified hot borate method significantly enhances the yield of high-quality RNA from cotton (Gossypium hirsutum L.). Anal. Biochem. 223:7-12.

Wang, Y., L.L. Georgi, G.L. Reighard, R. Scorza, and A.G. Abbott. 2002. Genetic mapping of the evergrowing gene in peach [Prunus persica (L.) Batsch]. Genetics 93:352-358.

Werner, D.J. and W.R. Okie. 1998. A history and description of the Prunus persica plant introduction collection. HortScience 33:787793.

Yamane, H., Y. Kashiwa, T. Ooka, R. Tao, and K. Yonemori. 2008. Suppression subtractive hybridization and differential screening reveals endodormancy-associated expression of an SVP/AGL24-type MADS-box gene in lateral vegetative buds of japanese apricot. J. Amer. Soc. Hort. Sci. 133:708-716.

Zhang, J. and M. Nei. 1996. Evolution of Antennapedia-class homeobox genes. Genetics 142:295-303. 